Published in final edited form as:

J Am Chem Soc. 2016 February 24; 138(7): 2130-2133. doi:10.1021/jacs.5b13180.

\title{
Photoinduced Electron Transfer Elicits a Change in the Static Dielectric Constant of a de Novo Designed Protein
}

\author{
Nicholas F. Polizzi ${ }^{a}, \S$, Matthew J. Eibling ${ }^{b, \S}$, Jose Manuel Perez-Aguilar ${ }^{b, \dagger}$, Jeff Rawson ${ }^{c}$, \\ Christopher J. Lanci ${ }^{\mathrm{b}}$, H. Christopher Fry ${ }^{\mathrm{b}, \boldsymbol{\uparrow}}$, David N. Beratan ${ }^{\mathrm{a}, \mathrm{c}, \mathrm{d}}$, Jeffery G. Saven ${ }^{\mathrm{b},}{ }^{*}$, and \\ Michael J. Therien ${ }^{\mathrm{c},}$, \\ aDepartment of Biochemistry, Duke University, Durham, North Carolina 27708, United States \\ bDepartment of Chemistry, University of Pennsylvania, Philadelphia, Pennsylvania 19104-6323, \\ United States \\ 'Department of Chemistry, Duke University, Durham, North Carolina 27708, United States \\ dDepartment of Physics, Duke University, Durham, North Carolina 27708, United States
}

\begin{abstract}
We provide a direct measure of the change in effective dielectric constant within a protein matrix after a photoinduced electron transfer (ET) reaction. A linked donor-bridge-acceptor molecule, $\mathrm{PZn}-\mathrm{Ph}-\mathrm{NDI}$, consisting of a (porphinato)Zn donor ( $\mathrm{PZn})$, a phenyl bridge $(\mathrm{Ph})$, and a naphthalene diimide acceptor (NDI), is shown to be a 'meter' to indicate protein dielectric environment. We calibrated PZn-Ph-NDI ET dynamics as a function of solvent dielectric, and computationally de novo designed a protein $S C P Z n I 3$ to bind PZn-Ph-NDI in its interior. Mapping the protein ET dynamics onto the calibrated ET catalogue shows that $S C P Z n I 3$ undergoes a switch in the effective dielectric constant following photoinduced ET, from $\varepsilon_{\mathrm{S}} \sim 8$ to $\sim 3$.
\end{abstract}

\section{Graphical abstract}

\footnotetext{
Corresponding Authors. saven@sas.upenn.edu, michael.therien@duke.edu.

đPresent Addresses

Argonne National Laboratory, Center for Nanoscale Materials, 9700 South Cass Avenue, Argonne, Illinois 60439, USA

†IBM Thomas J. Watson Research Center, Yorktown Heights, New York 10598, USA

$\S_{\text {These authors contributed equally. }}$

Supporting Information. Details regarding chemical synthesis, protein design and characterization, additional pump-probe spectra and methods, KIE data, a table of ET lifetimes of PZn-Ph-NDI in various solvents, parameters for theoretical modeling of dielectric dependence of ET lifetimes. This material is available free of charge via the Internet at http://pubs.acs.org.

The authors declare no competing financial interests.
} 


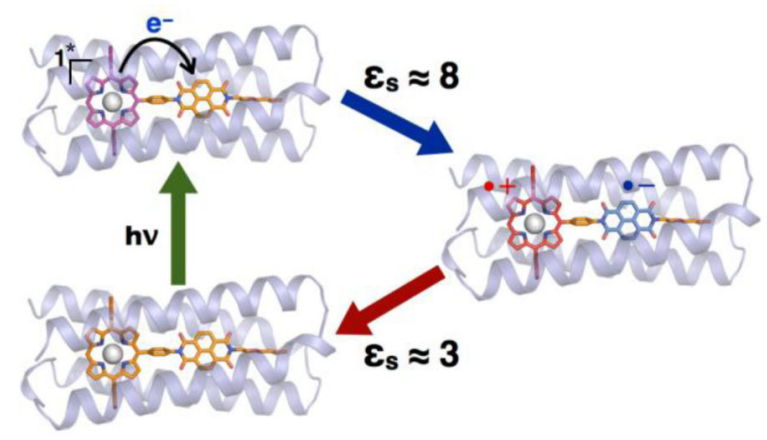

Anisotropic protein interiors are often described by an effective, uniform static dielectric constant $\left(\varepsilon_{\mathrm{s}}\right)$ of $2-15 .{ }^{1}$ The magnitude of $\varepsilon_{\mathrm{S}}$ influences critically biological electron-transfer (ET) reactions. ${ }^{2}$ Through its effect on the $\mathrm{pK}_{\mathrm{a}}$ of buried amino-acid residues, $\varepsilon_{\mathrm{S}}$ also impacts proton-coupled ET and proton-transfer processes. ${ }^{1 \mathrm{a}, 3}$ Theoretical and experimental studies of ET in proteins typically assume $\varepsilon_{\mathrm{s}}$ does not change as a result of the ET event. ${ }^{4}$ In this report, we provide direct evidence for a change in the protein $\varepsilon_{\mathrm{s}}$ following ET that occurs within a de novo designed protein matrix.

To interrogate dynamical changes in the magnitude of protein $\varepsilon_{\mathrm{s}}$ upon photoinduced charge separation, we utilize a donor-bridge-acceptor (DBA) molecule, ${ }^{5}$ with ET dynamics displaying marked sensitivity to: (i) solvent $\varepsilon_{\mathrm{s}}$; (ii) axial metal coordination; and (iii) deuteration of water (kinetic isotope effect). We calibrated these effects for the DBA molecule (5,15-diphenyl-10-(4'-[N-yl-(N'-(11-hydroxy-3,6,9-trioxoundecyl)naphthalene-1,4,5,8-tetracarboxylate diimide)]phenyl-porphinato)zinc(II) (PZn-Ph-NDI, Figure 1) in a variety of organic solvents, and computationally designed a tetra-a-helical protein $S C P Z n I 3$ to bind $\mathrm{PZn}-\mathrm{Ph}-\mathrm{NDI}$ in its interior.

PZn-Ph-NDI exhibits signature photoinduced ET dynamics following preparation of the PZn-localized $\mathrm{S}_{1}$ excited state, ${ }^{1}(\mathrm{PZn}) *$-Ph-NDI (Figure $\left.1 \mathrm{~A}\right)$. In the low $\varepsilon_{\mathrm{S}}$ solvent system 99:1 1,4-dioxane:N-methylimidazole (NMI), ${ }^{1}(\mathrm{PZn})^{*}$-Ph-NDI evolves to the chargeseparated state ${ }^{+\bullet} \mathrm{PZn}-\mathrm{Ph}-\mathrm{NDI}^{\bullet-}$ with a charge separation (CS) time of $\tau_{\mathrm{CS}}=12 \mathrm{ps}$. Charge recombination (CR) to the ground state is comparatively slow: $\tau_{\mathrm{CR}}=102 \mathrm{ps}$. The ET dynamics are tracked by the rise and subsequent decay of sharp spectral features centered at 480 and $610 \mathrm{~nm}$, characteristic of the $\mathrm{NDI}^{\circ-}$ radical anion. ${ }^{6}$ Species-associated difference spectra (Figure 1B), derived from a global fitting of the multi-wavelength transient absorption data to a sequential kinetic mechanism, also show clear signatures of the NDI ${ }^{\circ-}$ anion (red spectrum, prominent absorbance maxima at 480, 610, and $780 \mathrm{~nm}$ ).

Figure 2 displays the CS and CR time constants of PZn-Ph-NDI measured in organic solvents with $1 \%$ NMI. CR dynamics (red) depend strongly on $\varepsilon_{\mathrm{s}}$, with $\tau_{\mathrm{CR}}$ decreasing monotonically from 102 ps to 4 ps. CS dynamics (blue) show a $\tau_{\mathrm{CS}}$ minimum near $\varepsilon_{\mathrm{s}}=9$. Indeed, the observed trends for $\tau_{\mathrm{CS}}$ and $\tau_{\mathrm{CR}}$ are in agreement with those expected from the Marcus activated factor of ET rate theory ${ }^{2 \mathrm{a}, 7}$ (Figure 2, inset), using parameters appropriate for PZn-Ph-NDI (Supporting Information). While the ET dynamics of PZn-Ph-NDI displayed in Figure 2 approach the low ps time regime in some solvents, all time constants 
are well above solvent relaxation times, such that ET is not limited by dynamic solvent effects. ${ }^{8}$

NMI coordination to the PZn moiety accelerates CS and CR relative to dynamics evinced in neat solvents (Figure 2, purple, and Figure S3), resulting from the more negative oxidation potential of PZn when bound to the electron donating imidazole ligand. ${ }^{9}$ ET kinetics measured in solvents with $1 \%$ NMI provide a reference for comparison to kinetics measured within the protein environment of $S C P Z n I 3$, where $\mathrm{PZn}$ is coordinated by histidine.

Accounting for axial coordination effects allows us to isolate the $\varepsilon_{\mathrm{S}}$ contribution to the ET dynamics within the $S C P Z n I 3$ protein.

The $\tau_{\mathrm{CS}}$ of PZn-Ph-NDI exhibits a kinetic isotope effect (KIE) in 9:1 1,4-dioxane:H(D) ${ }_{2} \mathrm{O}$ mixtures (Figure 3). The $\mathrm{KIE}=\tau_{\mathrm{CS}}\left(\mathrm{D}_{2} \mathrm{O}\right) / \tau_{\mathrm{CS}}\left(\mathrm{H}_{2} \mathrm{O}\right)$ decreases from 1.4 to 1.2 upon NMI coordination to $\mathrm{PZn}$. Interestingly, although $\tau_{\mathrm{CR}}$ decreases with increasing water content, consistent with the dielectric dependence in Figure $2, \tau_{\mathrm{CR}}$ does not display a KIE (Table S1, Figures S4-S7). These observations suggest that CS is more tightly coupled than CR to water H-bond interactions with the imide carbonyls. ${ }^{10}$ The $\tau_{\mathrm{CS}} \mathrm{KIE}$ is an experimental probe of PZn-Ph-NDI water solvation within ground-state $S C P Z n I 3$.

To explore ET dynamics in a protein environment, the tetra-a-helical protein $S C P Z n I 3$ was computationally de novo designed ${ }^{11}$ to bind $\mathrm{PZn}-\mathrm{Ph}-\mathrm{NDI}$ in an interior consisting of hydrophobic amino acids (Figure 4 and Supporting Information). Electronic absorption and circular dichroism spectra of the complex in aqueous buffer confirm 1:1 binding of $\mathrm{PZn}-\mathrm{Ph}$ NDI to the interior of a helical protein and verify histidine coordination of the PZn moiety (Figure 4, B and C, Figures S21-S23). Thus the electronic environment of PZn-Ph-NDI is consistent with that of a protein interior.

The ET dynamics of PZn-Ph-NDI within the protein interior was monitored following electronic excitation of the PZn-Ph-NDI / SCPZnI3 complex in $\mathrm{D}_{2} \mathrm{O}$ buffer and analyzed via decay-associated difference spectra derived from global fitting of the multi-wavelength transient data (Figure 5). The CR dynamics of PZn-Ph-NDI in $S C P Z n I 3$ are bi-exponential: we assign the dominant CR time $\tau_{\mathrm{CR}}=22$ ps to the $S C P Z n I 3$ holo-dimer, and the CR time $\tau_{\mathrm{CR}}=69$ ps to the $S C P Z n I 3$ holo-monomer, as their respective amplitudes track with the approximate 2:1 dimer:monomer ratio determined from analytical gel filtration (Supporting Information). CS occurs in monomer and dimer with the same time constant $\tau_{\mathrm{CS}}=7.5 \mathrm{ps}$. The overlapping distributions of $\tau_{\mathrm{CS}}$ for $S C P Z n I 3 / \mathrm{H}(\mathrm{D})_{2} \mathrm{O}$ buffer (Figure 3) do not exhibit a KIE, indicating the lack of a significant role played by water in the CS event. On the basis of this observation, we conclude that PZn-Ph-NDI is sequestered from solvent within a wellpacked protein interior in both the dimer and monomer.

By comparison with Figure 2, each ET time constant exhibited by PZn-Ph-NDI can be mapped to an effective $\varepsilon_{\mathrm{s}}$. In the $S C P Z n I 3$ holo-monomer, $\mathrm{CS}$ and $\mathrm{CR}$ time constants $\left(\tau_{\mathrm{CS}}=\right.$ $7.5 \mathrm{ps}, \tau_{\mathrm{CR}}=69 \mathrm{ps}$ ) cannot be uniquely mapped to a single effective $\varepsilon_{\mathrm{S}}$ that jointly recovers both time constants. Two distinct $\varepsilon_{\mathrm{S}}$ values are needed (Figure 2, green). Accounting for the relative errors in the measured time constants (Supporting Information), as well as the nonmonotonic nature of the CS dependence on solvent dielectric, the range of the effective $\varepsilon_{\mathrm{s}}$ 
that describes CS ( $\tau_{C S}=7.5 \mathrm{ps}$ ) is 8.3 to 13.4. We take $\varepsilon_{S}{ }^{(\mathrm{CS})}=8.3$ as a lower limit. The effective $\varepsilon_{\mathrm{S}}$ describing $\mathrm{CR}$ is well defined $\left(\varepsilon_{\mathrm{S}}{ }^{(\mathrm{CR})}=3.0 \pm 0.1\right)$. Indeed, CS resembles that in 99:1 THF:NMI $\left(\varepsilon_{\mathrm{s}} \approx 8\right)$, whereas CR resembles that in 99:1dimethoxy-methane:NMI $\left(\varepsilon_{\mathrm{s}} \approx\right.$ $3)$. Importantly, these assignments do not depend on any underlying model: they result from mapping dynamical data acquired for the $S C P Z n I 3$ holoprotein onto an extensive body of analogous data obtained for PZn-Ph-NDI in widely varying dielectric environments. While a protein interior is not a uniform dielectric material, these $\varepsilon_{S}$ values reflect the effective dielectric probed by PZn-Ph-NDI buried in the $S C P Z n I 3$ interior, both pre- and post-charge separation.

How can the protein achieve such changes in dielectric on these fast $(<100 \mathrm{ps})$ ET time scales? These results suggest a rigidification of the protein sidechains upon formation of the ${ }^{+\bullet} \mathrm{PZn}-\mathrm{Ph}-\mathrm{NDI}^{{ }^{-}-} \mathrm{CS}$ state. Interestingly, the reduction in $\varepsilon_{\mathrm{S}}$ after the ET event suggests little solvational contribution to the CS state from polar water, despite the protein's small size, and contrary to commonly accepted views. ${ }^{1 \mathrm{~b}}$ The dielectric constant is a key parameter affecting CS and CR rates in biological proteins. A $\varepsilon_{S}$ switching mechanism may warrant a reexamination of the energetic and electronic parameters derived from measurements of biological ET rates. Dielectric switching may provide a means to independently manipulate forward and back ET rates in natural catalytic proteins and artificial photosynthetic devices. Indeed, the ET time scales of PZn-Ph-NDI are similar to that of the primary ET in photosynthesis; ${ }^{12}$ a decrease in protein $\varepsilon_{S}$ upon the primary CS event would further slow wasteful inverted-regime $\mathrm{CR}$ in the photosynthetic reaction center.

\section{Supplementary Material}

Refer to Web version on PubMed Central for supplementary material.

\section{Acknowledgments}

This work was supported through grants from the National Institutes of Health (R01 GM-071628) and the National Science Foundation (CHE-1413333).

\section{REFERENCES}

1. (a) Alexov EG, Gunner MR. Biochemistry. 1999; 38:8253-8270. [PubMed: 10387071] (b) Schutz CN, Warshel A. Proteins: Struct. Funct. Bioinform. 2001; 44:400-417.(c) Isom DG, Castañeda CA, Cannon BR, Velu PD, García-Moreno EB. Proc. Natl. Acad. Sci. USA. 2010; 107:16096-16100. [PubMed: 20798341] (d) Kukic P, Farrell D, McIntosh LP, García-Moreno EB, Jensen KS, Toleikis Z, Teilum K, Nielsen JE. J. Am. Chem. Soc. 2013; 135:16968-16976. [PubMed: 24124752]

2. (a) Marcus RA, Sutin N. BBA-Rev. Bioenerg. 1985; 811:265-322.(b) Steffen MA, Lao K, Boxer SG. Science. 1994; 264:810-816. [PubMed: 17794722]

3. (a) Hammes-Schiffer S, Stuchebrukhov AA. Chem. Rev. 2010; 110:6939-6960. [PubMed: 21049940] (b) Glover SD, Jorge C, Liang L, Valentine KG, Hammarström L, Tommos C. J. Am. Chem. Soc. 2014; 136:14039-14051. [PubMed: 25121576] (c) Migliore A, Polizzi NF, Therien MJ, Beratan DN. Chem. Rev. 2014; 114:3381-3465. [PubMed: 24684625]

4. (a) Warshel A, Papazyan A. Curr. Opin. Struct. Biol. 1998; 8:211-217. [PubMed: 9631295] (b) Gray HB, Winkler JR. Proc. Natl. Acad. Sci. USA. 2005; 102:3534-3539. [PubMed: 15738403] (c) Li L, Li C, Zhang Z, Alexov E. J. Chem. Theory Comput. 2013; 9:2126-2136. [PubMed: 23585741]

5. Redmore NP, Rubtsov IV, Therien MJ. J. Am. Chem. Soc. 2003; 125:8769-8778. [PubMed: 12862471] 
6. Gosztola D, Niemczyk MP, Svec W, Lukas AS, Wasielewski MR. J. Phys. Chem. A. 2000; 104:6545-6551.

7. Hopfield, JJ. Protein Structure: Molecular and Electronic Reactivity. Austin, R.; Buhks, E.; Chance, B.; Dutton, P.; De Vault, D.; Frauenfelder, H.; Gol'danskii, V., editors. New York: Springer; 1987. p. 167-185.

8. (a) Kahlow MA, Kang TJ, Barbara PF. J. Phys. Chem. 1987; 91:6452-6455.(b) Maroncelli M, MacInnis J, Fleming GR. Science. 1989; 243:1674-1681. [PubMed: 17751278]

9. Kadish KM, Shiue LR, Rhodes RK, Bottomley LA. Inorg. Chem. 1981; 20:1274-1277.

10. (a) Buhks E, Bixon M, Jortner J. J. Phys. Chem. 1981; 85:3763-3766.(b) Shirota H, Pal H, Tominaga K, Yoshihara K. J. Phys. Chem. A. 1998; 102:3089-3102.

11. (a) Bender GM, Lehmann A, Zou H, Cheng H, Fry HC, Engel D, Therien MJ, Blasie JK, Roder H, Saven JG, Degrado WF. J. Am. Chem. Soc. 2007; 129:10732-10740. [PubMed: 17691729] (b) Calhoun JR, Kono H, Lahr S, Wang W, DeGrado WF, Saven JG. J. Mol. Biol. 2003; 334:11011115. [PubMed: 14643669] (c) Cochran FV, Wu SP, Wang W, Nanda V, Saven JG, Therien MJ, DeGrado WF. J. Am. Chem. Soc. 2006; 128:663-663.(d) Fry HC, Lehmann A, Saven JG, DeGrado WF, Therien MJ. J. Am. Chem. Soc. 2010; 132:3997-4005. [PubMed: 20192195] (e) Fry HC, Lehmann A, Sinks LE, Asselberghs I, Tronin A, Krishnan V, Blasie JK, Clays K, DeGrado WF, Saven JG, Therien MJ. J. Am. Chem. Soc. 2013; 135:13914-13926. [PubMed: 23931685]

12. (a) Carter B, Boxer SG, Holten D, Kirmaier C. Biochemistry. 2009; 48:2571-2573. [PubMed: 19245209] (b) Heller BA, Holten D, Kirmaier C. Science. 1995; 269:940-945. [PubMed: 7638616] (c) Wasielewski MR, Johnson DG, Seibert M, Govindjee. Proc. Natl. Acad. Sci. USA. 1989; 86:524-528. [PubMed: 16594012] 


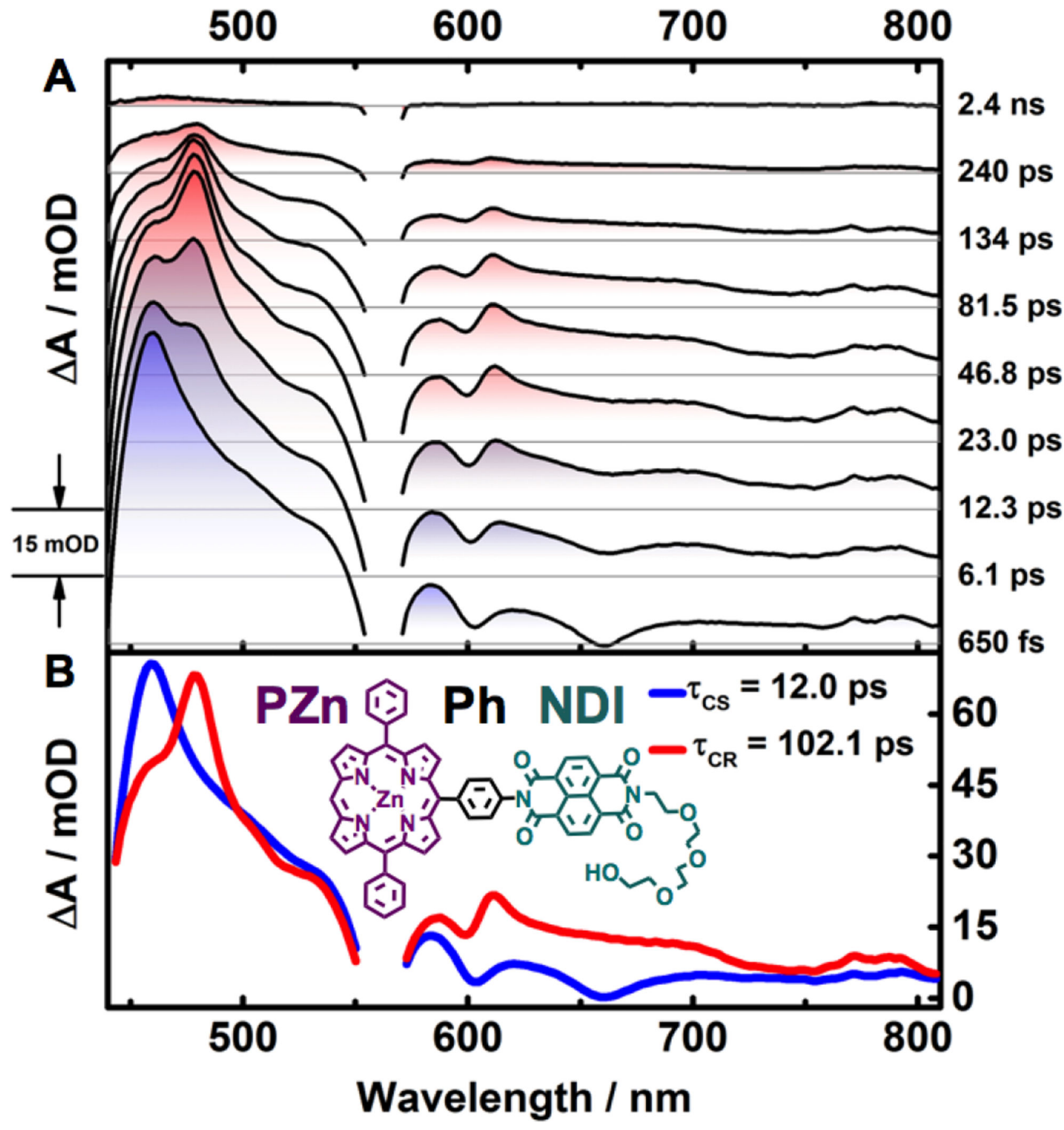

Figure 1.

Pump-probe transient dynamics of PZn-Ph-NDI in 99:1 1,4-dioxane:N-methylimidazole. (A) Transient dynamical data at various pump-probe time delays, which are labeled on the right. Each spectrum is offset by $15 \mathrm{mOD}$ for clarity. (B) Species-associated difference spectra (sans laser scatter at $560 \pm 5 \mathrm{~nm}$ ) with associated ET time constants. Experimental conditions: $\lambda_{\mathrm{ex}}=560 \mathrm{~nm}, \mathrm{P}_{\mathrm{ex}}=850 \mathrm{~nJ} /$ pulse, $\mathrm{T}=21^{\circ} \mathrm{C}$, magic angle polarization. 


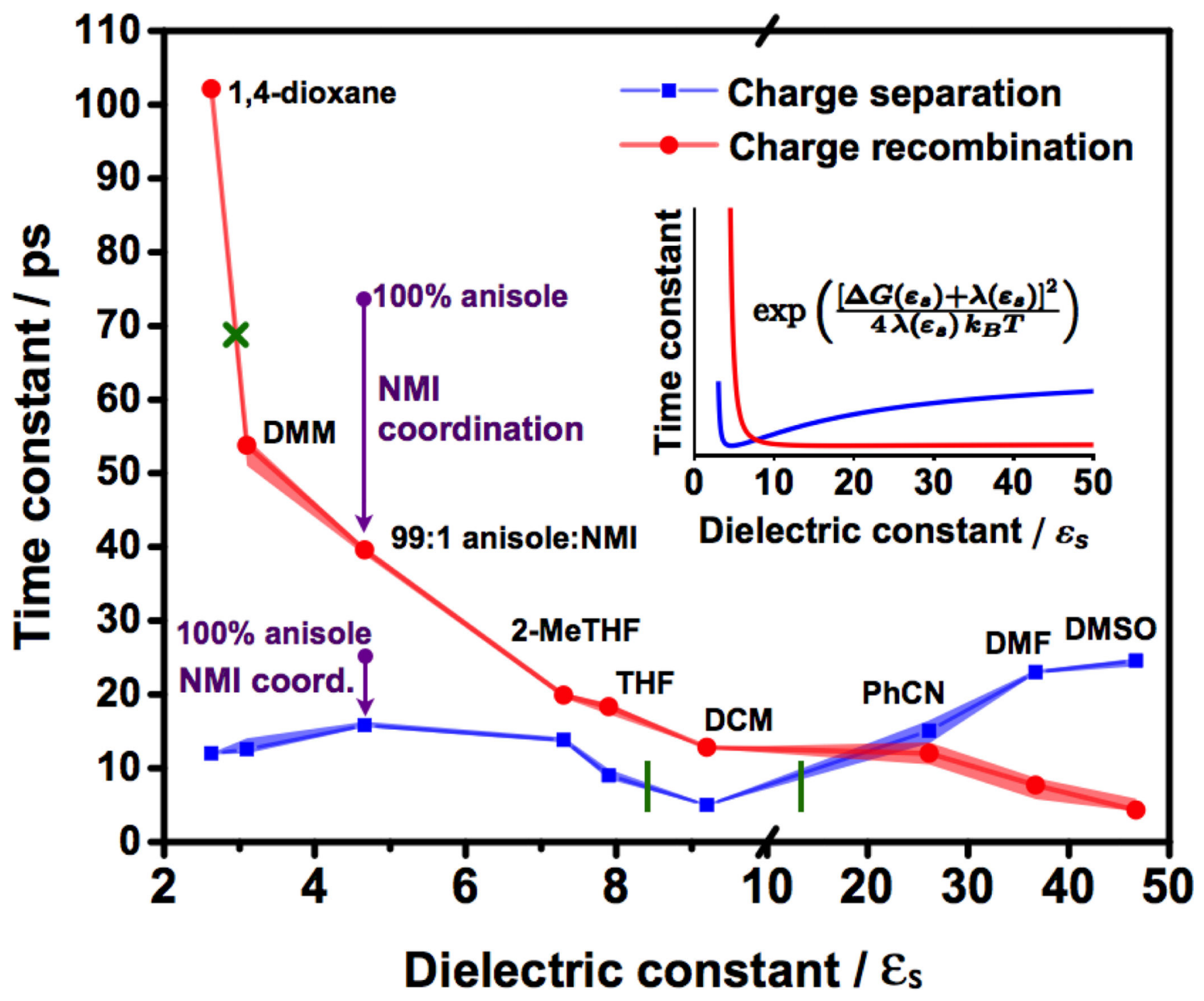

Figure 2.

ET time constants $\tau_{\mathrm{CS}}$ and $\tau_{\mathrm{CR}}$ of PZn-Ph-NDI as a function of solvent static dielectric constant $\left(\varepsilon_{\mathrm{S}}\right)$. All solvents include $1 \% \mathrm{~N}$-methylimidazole (NMI). $\varepsilon_{\mathrm{S}}$ is a volume-fraction weighted sum of component $\varepsilon_{\mathrm{s}}$ values. Shaded line widths indicate confidence intervals of the fitted time constants (bounded at $16 \%$ and $84 \%$ percentiles of the bootstrapped lifetime distributions). For the $S C P Z n I 3$ holo-monomer, $\tau_{\mathrm{CR}}$ is denoted with a green $\mathrm{X}$. Green vertical lines mark the range of dielectric constants consistent with the measured value of $\tau_{\mathrm{CS}}$ in the $S C P Z n I 3$ holo-monomer. Purple arrows display an exemplary reduction of ET time constant magnitudes that occur upon NMI coordination, shown explicitly for anisole solvent. Inset: expected dielectric dependence of $\tau_{\mathrm{CS}}$ (blue) and $\tau_{\mathrm{CR}}$ (red) according to ET rate theory (see Supporting Information). $\mathrm{DMM}=$ dimethoxymethane, $\mathrm{THF}=$ tetrahydrofuran, 2-MeTHF = 2-methylTHF, DCM = dichloromethane, $\mathrm{PhCN}=$ benzonitrile, $\mathrm{DMF}=\mathrm{N}, \mathrm{N}$-dimethylformamide, $\mathrm{DMSO}=$ dimethylsulfoxide . 


\section{SCPZn/3}

$\mathrm{KIE}=1 \mid \quad$ 99:1 1,4-dioxane:NMI

$\mathrm{KIE}=1.2$

Figure 3.

Distributions of CS time constant $\tau_{C S}$ displayed by PZn-Ph-NDI within various solvation environments. Distributions are derived from bootstrapping the residuals of the global fit of the pump-probe data (see Supporting Information). Measurements in 1,4-dioxane contain $10 \% \mathrm{H}_{2} \mathrm{O}$ or $\mathrm{D}_{2} \mathrm{O}$. Measurements with $S C P Z n I 3$ were carried out in $50 \mathrm{mM} \mathrm{NaPi} / 150 \mathrm{mM}$ $\mathrm{NaCl}$ buffer with $100 \% \mathrm{H}_{2} \mathrm{O}$ or $\mathrm{D}_{2} \mathrm{O}$. Confidence intervals in gray display $16 \%$ and $84 \%$ percentiles of the bootstrapped lifetime distributions. The optimum time constant is shown as the middle, vertical line. Black lines overlaying the bootstrap histograms are Gaussian fits to the distributions. 

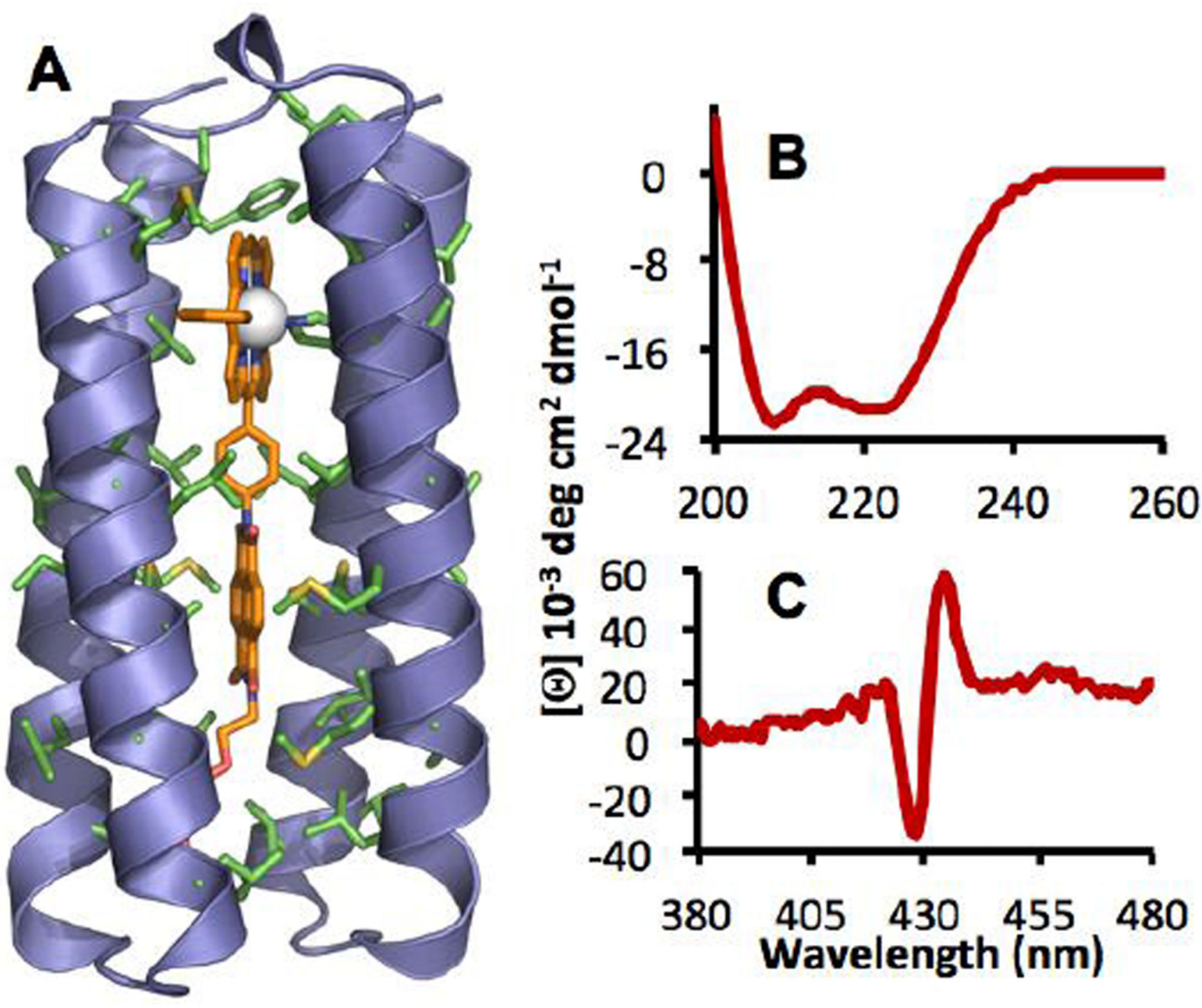

Figure 4.

(A) Model of $S C P Z n I 3$ showing interior hydrophobic residues (green) surrounding $\mathrm{PZn}-\mathrm{Ph}$ NDI (orange). (B, C) Circular dichroism spectra of SCPZnI3 holoprotein. (B) Molar ellipticity per residue is consistent with a helical structure. (C) Molar ellipticity (Cotton effect) in visible region indicates achiral cofactor resides in a structured, chiral environment (see Figure S21). 


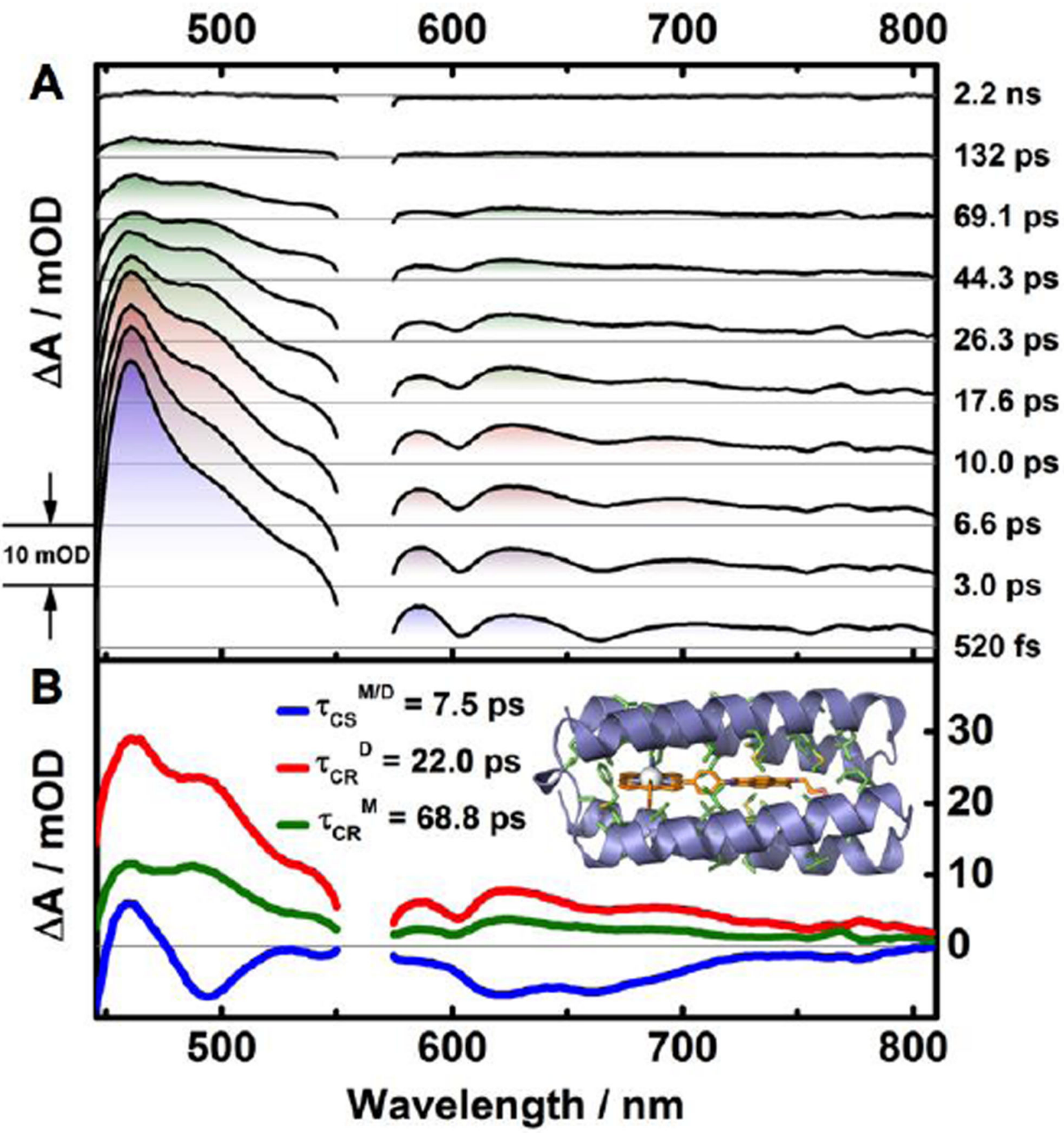

Figure 5.

Pump-probe transient dynamics of PZn-Ph-NDI / $S C P Z n I 3$ complex in $\mathrm{D}_{2} \mathrm{O}$ buffer. (A) Transient dynamical data at pump-probe time delays labeled on the right. Each spectrum is offset by $10 \mathrm{mOD}$ for clarity. (B) Decay-associated difference spectra (sans laser scatter at $560 \pm 5 \mathrm{~nm}$ ) with associated $\tau_{\mathrm{CR}}$ and $\tau_{\mathrm{CS}}$. D and $\mathrm{M}$ superscripts refer to dimer and monomer, respectively. Experimental conditions: $\lambda_{\mathrm{ex}}=560 \mathrm{~nm}, \mathrm{P}_{\mathrm{ex}}=920 \mathrm{~nJ} /$ pulse, $\mathrm{T}=21^{\circ} \mathrm{C}$, magic angle polarization. 